American Journal of Pharmacology and Toxicology 3 (2): 201-208, 2008

ISSN 1557-4962

(C) 2008 Science Publications

\title{
Evaluation of the Lack of Essential Information in Medical Literature
}

\author{
${ }^{1}$ Muaed J. Alomar, ${ }^{2}$ Anas A. Hourani, ${ }^{2}$ Syed A. Sulaiman and ${ }^{3}$ Claire C. Strauch \\ ${ }^{1}$ Department of Clinical Pharmacy, Faculty of Pharmacy P.O. Box 7826 Fujairah, \\ United Arab Emirates \\ ${ }^{2}$ University Sians Malaysia (USM), Penang State, Malaysia \\ ${ }^{3}$ Ajman University of Science and Technology Network, Fujairah, UAE
}

\begin{abstract}
Primary literature is a basic tool for medical knowledge and for future research. Every researcher relies at some point on the information available in published literature. Also, primary literature is utilized to develop guidelines for the medical use of medications in all kinds of diseases, accuracy is a must in this regard. To evaluate essential information regarding patients, drugs and diseases omitted from efficacy literature on antihypertensive drug safety. Data on antihypertensive adverse drug reactions we retrospectively collected from 219 medical articles using search engines IDIS Iowa Drug Information Services, ScienceDirect, Medscape, Pubmed, Ebscohost, Inside web, Proquest, Emerald and Medline. The data for the study was collected from primary literature carried out on antihypertensive medications in previous years. All of the available information regarding patients (patient's factors) and drugs was collected in order to evaluate the percentage of the omitted data. 219 primary journal articles were collected, 640 medications from these articles were analyzed. Some of the 640 medications are the same but in different articles. Out of 640 medications collected, 15718 data entries were used which represent patient's factors and drug factors, age, gender, type of job, renal conditions, alcohol consumption, use of other medications, other diseases, drug dose, drug frequency, dosage form. All these factors were evaluated for the effect on developing adverse drug reactions. The omitted data was classified into four different categories. Age, gender, race, smoking, health status, medication use and any other information related to the patients is basic information which needs to be available in all literature, not just to be mentioned in the methodology and sampling, but to be specified carefully and fully in the results.
\end{abstract}

Key words: Omitted, patient's factors, missing data, information technology, drug safety

\section{INTRODUCTION}

Primary literature is a basic tool for medical knowledge and for future research. Every researcher relies at some point on the information available in published literature. Also, primary literature is utilized to develop guidelines for the medical use of medications in all kinds of diseases, accuracy is a must in this regard. Writing literature in a scientific way is mandatory, certain information however and its availability should be also mandatory. The use of evidence based medicine is becoming a basic tool in ensuring good medical outcomes. Because primary literature provides us with a certain level of accuracy, it is sometimes accepted as fact. Evidence based medicine requires we examine the validity, type and the amount of information provided by this literature.

In medical practice, our decision depends totally on literature. For that reason, primary literature needs further evaluation. The conditions might need reviewing or better organization needed. By examining the available literature it is evident how different each one is, in the method of writing, the availability of basic data, the results and their accuracy and in the unprovided data. The core of the study is to evaluate whether any essential, decision making data has been omitted.

Objectives: To evaluate essential information regarding patients, drugs and diseases omitted from efficacy literature on antihypertensive drug safety.

\section{MATERIALS AND METHODS}

Data on antihypertensive adverse drug reactions was retrospectively collected from 219 medical articles using search engines IDIS Iowa Drug Information Services, Science Direct, Medscape, Pubmed,

Corresponding Author: Muaed J. Al Omar, Department of Clinical Pharmacy, Faculty of Pharmacy P.O. Box 7826 Fujairah, United Arab Emirates (UAE) Tel: 00971507157641 Fax: 0097192223176 
Ebscohost, Inside web, Proquest, Emerald and Medline. 640 antihypertensive medications were evaluated from the 219 medical articles. It is important to note that the percentage of omitted data will be evaluated for the 640 medications since each medication is considered a different identity.

Data collection: The data for the study was collected from primary literature carried out on antihypertensive medications in previous years.

All of the available information regarding patients (patient's factors) and drugs was collected in order to evaluate the percentage of the omitted data.

Patient's factors: During data collection, all of the possible differences among patients were termed patient's factors. These include, Age, Gender, Race, Diseases, Creatinine Clearance, Allergy, Drinking alcohol, Fetus age, Heart rate, Job, Maternity, Medications, Obesity, Smoking, Time of taking the medication (dosing), Onset of ADR, Treatment period and Weight.

These factors differentiate between patients and could be the reason why some patients develop adverse drug reactions towards certain medication and others do not.

Data regarding the availability of this information in medical literature is evaluated in which each article is screened for the lack of these factors.

This research will focus on the following antihypertensive drugs, Angiotensin converting enzyme inhibitors (Captopril, Enalapril, Lisinopril, Imidapril, Benazepril, Trandolapril, Zofenopril, Ramipril, Cilazapril, Quinapril, Delapril, Fosinopril, Perindopril, Moixipril), Angiotensin II antagonists (Valsartan, Candesartan, Eprosartan, Irbesartan, Losartan, Telmisartan, Olmesartan, Tasosartan), Calcium channel blockers (Amlodipine, Barnidipine, Diltiazem, Felodipine, Isradipine, Lacidipine, Lercanidipine, Mandipine, Mibefradil, Nifedipine, Nifedipine CR, Verapamil, Rilmenidine, Nifedipine GITS, Nisoldipine), Beta blockers (Atenolol, Betaxolol, Bisoprolol, carvedilol, Labetalol, Metoprolol, Nebivolol), Diuretics (Chlorthalidone, Hydrochlorothiazide, Hydrochlorothiazide+amiloride, Indapamide, Indapamide SR), Alpha blockers (Doxazosin, Doxazosin Terazosin, Tetrazosin, GITS, Hydralazine), Mineralocorticoid receptor antagonists (Eplerenone), Peripheral vasodilators (Pinacidil).

The collection of data was carried out in a systematic manner using IDIS as a search method. The studies used from IDIS are the ones published from year 1989 to year 2006. In Medline and Pubmed, a search of abstracts was performed. If an abstract suited the subject of interest, the full study was taken either by direct order through the internet or by using other authorized search engines through Universiti Sians Malaysia's electronic library.

The following criteria was used for data collection and entry which includes: Name of the study, publication date, Category, Factor, Factor Patient No, Drug, Drug Dose, Dose Frequency, Dosage Form, Brand Name, ADR and Patient ADR No.

About 640 medications were collected from 219 different articles, the following data was collected for each medication, name of the medication, dose of the study, frequency of the dose, factors related to the patients and the drugs, adverse drug reaction related to each patient's or drug's factor, number of adverse drug reactions.

Patient related factors are, age, alcohol consumption, fetal age (for pregnancies), gender, job, Body mass index BMI or obesity, race and smoking.

Drug related factors are, Drug Dose, Dose Frequency, Dosage Form and Brand Name. For clarification show Fig. 1.

The omitted data which requires analyzing is of different types, some data is not mentioned at all in the study, for example, the number of ADR patients for a specific medication and specific category. Another type is the lack of unspecific data within a category, for example, age mentioned in the literature is 20-70 years of age. This range is wide and it includes the young, middle aged and elderly. This is misleading because different ages have different reactions to medications.

There are two main reasons for the publication of studies. First, using them for medical decision making which affects human life and well being. Secondly, as the building blocks for further research. Both of these situations require that basic data is available, because without it any decisions made will be misleading or may lead to an endless series of mistakes. The collected data was analyzed using Microsoft access.

\section{RESULTS}

About 219 primary journal articles were collected, 640 medications from these articles were analyzed. Some of the 640 medications are the same but in different articles. Out of 640 medications collected, 15718 data entries were used which represent patient's factors and drug factors, age, gender, type of job, renal conditions, alcohol consumption, use of other medications, other diseases, drug dose, drug frequency, dosage form. All these factors were evaluated for the effect on 
Am. J. Pharm. \& Toxicol., 3 (2): 201-208, 2008

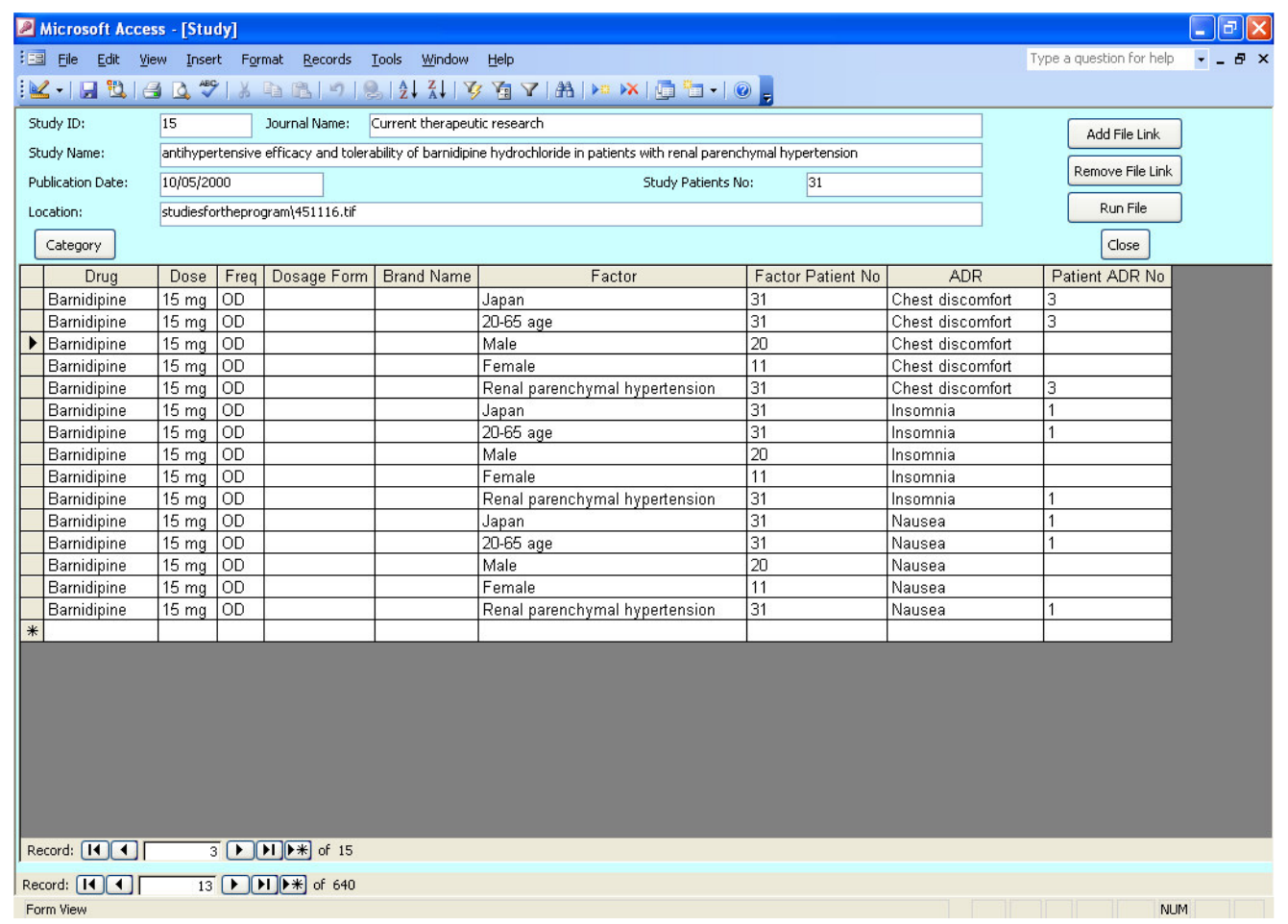

Fig. 1: Example for data entry using Microsoft access

developing adverse drug reactions. The omitted data was classified into four different categories.

The total amount of data collected for the ADRs is 15718. The total amount of omitted data is 5267 and the percentage of omitted data from the total is $35.41 \%$.

Types of missing data: A. Missing data: Data not provided by the articles. This is essential data which must be provided by the articles in order for the literature to be utilized properly. This data is Sub divided into three categories:

\section{- Patient related information}

Gender: Gender is very important in the occurrence of adverse drug reactions. Males and females are different in many aspects and this difference has a strong impact on the occurrence of adverse drug reactions. By not providing details of how many of the patients were male or female this can lead to confusing data. $91 \%$ of female and $92 \%$ of male are not specified for a specific ADR. In addition to that, $33 \%$ of the data regarding gender is not specified.

Some studies have shown that gender plays a role in the effect on adverse drug reaction, a study of sex differences in adverse reactions to antiretroviral drugs indicates a potential sex differences in the frequency and severity of adverse reactions to antiretroviral drugs $^{[1]}$.

In a north Indian study of Angiotensin converting enzyme inhibitors and cough, females had a higher incidence of cough as compared to males $37.9 \%$ versus $15.5 \%{ }^{[2]}$.

The difference in the occurrence of adverse drug reactions between male and female urges the authors of the research to provide this important information.

When adverse drug reactions are mentioned, all of the factors regarding the patient must be mentioned for optimum utilization of the literature.

Smoking: The percentage of missing data is $91 \%$. Which means that $91 \%$ of the data used in the study did not specify whether the patients who developed adverse drug reactions were smokers or non smokers.

Often a study mentions that a certain number of patients enrolled in the study are smokers. However, when adverse drug reactions are noted, they fail to mention whether the people who developed the adverse drug reactions were the smokers, non smokers or the ex-smokers. Medical practitioners cannot draw a medically useful conclusion about the adverse drug reaction if they do not know whether the adverse drug 


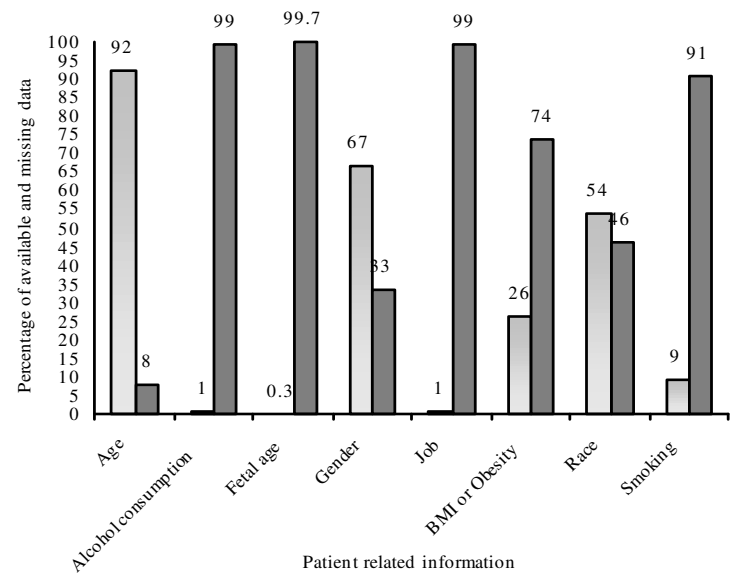

口PAIS \%: Percentage of the available in formation 口PMD \%: Percentage of missing data

Fig. 2: Missing data regarding patient related information

Table 1: Missing data regarding patient related information

\begin{tabular}{llll}
\hline Patient related info & NMPIM & PAIS\% & PMD\% \\
\hline Age & 588 & 92 & 8 \\
Alcohol consumption & 6 & 1 & 99 \\
Fetus age & 2 & 0.3 & 99.7 \\
Gender & 430 & 67 & 33 \\
Job & 1 & 1 & 99 \\
BMI or Obesity & 165 & 26 & 74 \\
Race & 347 & 54 & 46 \\
Smoking & 56 & 9 & 91 \\
\hline
\end{tabular}

NMPIM: Number of medications where patient information is mentioned. Knowing that the total number of medications included in this study is 640. PAIS: percentage of the available information. PMD: percentage of missing data.

Table 2: Missing data regarding drug related information

\begin{tabular}{lll}
\hline Drug related info & $\begin{array}{l}\text { No. of } \\
\text { missing data }\end{array}$ & $\begin{array}{l}\text { Percentage of } \\
\text { missing data }\end{array}$ \\
\hline Missing Drug Dose & 143 & 22.3 \\
Missing Dose Frequency & 142 & 22.2 \\
Missing Dosage Form & 612 & 96 \\
Missing Brand Name & 636 & 99.4 \\
\hline
\end{tabular}

reaction occurred because of the medication, the smoking, a combination of smoking and the medication or smoking withdrawal reactions. Many important questions need to be answered. Studies should provide this basic information in Table 1.

Drug related information: About $22.3 \%$ of drug doses are not provided, $22.2 \%$ of dose frequencies are not provided, $96 \%$ of drug dosage form is missing and $99.4 \%$ of drugs were not provided under their brand names show in Table 2.

Percentage of ADR missing data: About $35.4 \%$ of adverse drug reaction data were not provided which

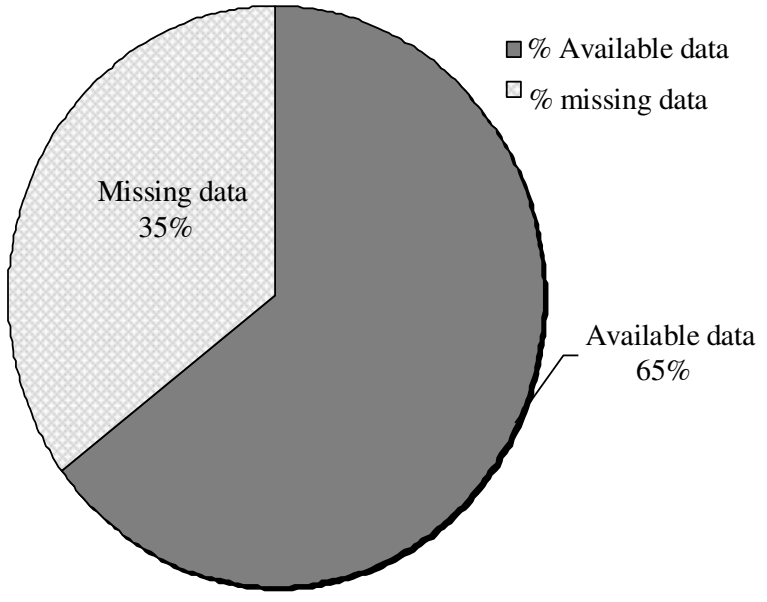

Fig. 3: Missing data regarding ADR related information

Table 3: Missing data regarding ADR related information

\begin{tabular}{llll}
\hline $\begin{array}{l}\text { Patient ADR } \\
\text { No. count }\end{array}$ & Total & $\begin{array}{l}\text { Missing patient } \\
\text { ADR No }\end{array}$ & $\begin{array}{l}\text { Percentage missing } \\
\text { patient ADR No }\end{array}$ \\
\hline 10151 & 15718 & 5567 & 35.4 \\
\hline
\end{tabular}

Table 4: Age description inaccuracy

\begin{tabular}{lllll}
\hline $\begin{array}{l}\text { Category } \\
\text { count }\end{array}$ & Factor & $\begin{array}{l}\text { Factor } \\
\text { count }\end{array}$ & $\begin{array}{l}\text { Total factor } \\
\text { count }\end{array}$ & $\begin{array}{l}\text { Percentage } \\
\text { factor }\end{array}$ \\
\hline Age & $>65$ age & 99 & 3094 & 3.2 \\
Age & $>75$ age & 33 & 3094 & 1.0 \\
Age & 10s year & 22 & 3094 & 0.7 \\
Age & $20-65$ age & 101 & 3094 & 3.3 \\
Age & 20-70 age & 972 & 3094 & 31.4 \\
Age & 40-81 age & 259 & 3094 & 8.4 \\
Age & 6-19 years & 3 & 3094 & 0.1 \\
Age & Adult & 6 & 3094 & 0.2 \\
Age & Geriatric & 79 & 3094 & 2.6 \\
\hline
\end{tabular}

means that from the 15718 patients from the studies, the ADR for 5567 were not provided as direct missing data show in Table 3

\section{Description inaccuracy}

Age distribution inaccuracy: Age is a very important factor which affects drug action, dosing, compliance and toxicity. Different age groups have different ways of response towards medications.

Table 4 provides the number of each age group mentioned in the literature and its relation to other age groups. The age ranges are varied, some are wide like $20-70$ years old and some are not specific like $<60$ years old.

The percentage of age group 20-70 years old is $31.42 \%$ (972/3094). The percentage of age group 20-65 years old is $3.26 \%$ (101/3094). The percentage of age group $40-81$ is $8.37 \%$ (259/3094). The percentage of age group $<60$ years old is $0.13 \%$ (4/3094). The 
percentage of age group $>65$ years old is $3.2 \%$ (99/3094). The percentage of age group 6-19 years old is $0.1 \%$ (3/ 3094). And the percentage of age group <20 years old is $2.17 \%(67 / 3094)$. The total percentage of all wide range age groups is $48.47 \%$ (1505/3094).

\section{DISCUSSION}

About $48.47 \%$ of the age data was in a wide range, this means that no specific age group can be considered and correlated with the response and toxicity of the medication involved in the study. Old people react differently towards medications than young people. Providing more accurate and precise data is valuable in this matter because it allows the user of the study to extract precise and useful information which can be utilized properly in the decision making process of pharmacotherapy and drug selection.

Elderly patients with multiple medical problems who are taking multiple medications, those who have a history of adverse drug reactions and those with a reduced capacity to eliminate medications are at high risk for adverse drug reactions.

All medications can produce ADRs, but not all patients develop the same level and type of ADRs, age is one of the very important factors that affect adverse drug reaction occurrence.

When studies say the age is less than 20 years, this includes newborn, infants, children and teenagers. These groups are completely different when it comes to drug function and toxicity.

Infants and very young children are at high risk of adverse drug reactions because their capacity to metabolize drugs is not fully developed.

Older people are at high risk of having an adverse drug reaction for several reasons. They are likely to have many health problems and thus to be taking several prescription and over the counter drugs. As people get old, the liver loses the ability to metabolize drugs ${ }^{[3]}$.

Older people tend to take more drugs than younger people because they are more likely to have several, often chronic disorders. On average, an older person takes four or five prescription drugs and two over-thecounter drugs each day. Also, older people are more than twice as susceptible to side effects of drugs as younger people ${ }^{[4]}$.

As people age, the amount of water in the body decreases and the amount of fat tissue relative to water, increases. Thus, in older people, drugs that dissolve in water reach higher concentrations because there is less water to dilute them and drugs that dissolve in fat accumulate more because there is relatively more fat tissue to store them. Also, as people age, the kidneys are less able to excrete drugs into the urine and the liver is less able to metabolize many drugs. Jimmy Jose and Padma G.M. Rao ${ }^{[5]}$ in their study have concluded that the incidence of ADRs among elderly adults and older adults were significantly higher than other age groups. They also elaborated that the type of ADR is different among age groups, type $\mathrm{A}$ reactions were more common among elderly adults $(85.9 \%)$ and type B reactions were more common in adults $(35 \%)$ compared to other age groups.

Because of all these age-related changes, many drugs tend to stay in an older person's body much longer than they would in a younger person's body, prolonging the drug's effect and increasing the risk of side effects.

Thus age and sex differences may contribute in the development of adverse drug reactions. In general practice in England, suspected adverse drug reactions to newly marketed drugs are recorded more often in adults aged between 30 and 59 years of age and are $60 \%$ more common in women than in men. The sex difference occurs in all age groups over 19 years of age ${ }^{[6]}$.

A study about the incidence and preventability of adverse drug events among older persons in the ambulatory setting concluded that adverse drug events are common and often preventable among older persons in the ambulatory clinical setting. More serious adverse drug events are more likely to be preventable. Prevention strategies should target the prescribing and monitoring stages of pharmaceutical care. Interventions focused on improving patient adherence with prescribed regimens and monitoring of prescribed medications ${ }^{[7]}$.

These studies emphasize the importance of age in determining adverse drug reactions and the degree of susceptibility, to the occurrence of these unwanted effects. This makes age a very important factor. In table 3 the age groups provided by literature are not categorized in a way which makes it easy to use. The range 20-65 years of age accounts for nine hundred and seventy two (972) out of the total age groups mentioned which is three thousand and ninety four (3094), the percentage is $31.42 \%$. This means that $31.42 \%$ of the age mentioned is not specific covering a very wide range, including young people, middle aged people and elderly people. It is documented in literature that old people are more susceptible to adverse drug reaction than the young. Other age groups mentioned which also not specific are 20-65 years old which accounts for one hundred and one (101) out of three thousand and ninety four (3094) with a percentage of 3.26\%. 67 (2.17\%) are $<20$ years old. $4(0.13 \%)$ are $<60.259(8.37 \%)$ are in the age of 40-81 years old. These results might be 
Am. J. Pharm. \& Toxicol., 3 (2): 201-208, 2008

misleading when used in the medical practice. Specific and clear age groups should be provided when each study discusses adverse drug reactions or safety and tolerability in order to make it useful for other researches to extract the correct data and use it in the right place. The total percentage of all wide range age groups is $48.47 \%$ ( 1505 out of 3094 ), this percentage is almost half of the data.

Race distribution inaccuracy: The current dictionary definition of race is a family, tribe, people, or nation belonging to the same common stock, or a class or kind of people unified by shared interests, habits, or characteristics ${ }^{[8]}$. In most medical settings patients self determine their racial origin ${ }^{[9]}$, which gives them ample chance to mislead medical professionals. In the 2000 census in the United States, 1 in 4 individuals in some regions of the United States chose more than 1 box indicating race and ethnicity, which means that people are not fully aware of the racial classifications or there is no clear classification for race. In the United States there is approximately 126 categories of race ${ }^{[9]}$. This confusion needs from the researchers to concentrate on finding ways to categorize race and ethnicity in away to be flexible to evolve with changing time and environmental factors, migration, food and other related effectors.

Risch $e t a l{ }^{[10]}$ argued that five major racial groups can be identified (Africans, Caucasians, Pacific Islanders, Asians and Native Americans). But human races are mixed and there is no chance that it can be controlled especially with migrations and travelling.

A dark-skinned, curly-headed person who identifies as African American may, indeed, have much in his or her history and upbringing to justify that identification. But he or she may also have a white grandparent and several Cherokee ancestors

In Table 5 the race number for some studies reflects the country where the study is carried out; others mention the race of each patient group.

Table 5 reflects the mess in providing data, which shows the lack of specific categorization approaches for the race.

Race and ethnicity are crucial in medical practice, According to Sarah ${ }^{[11]}$ there is a big difference in developing adverse drug reactions for cardiovascular medications among different ethnic and race groups which means it is a determining factor in the treatment of individual patients.

When medical literature is not providing the race and mentions only the country where the study is carried out, many countries included in this study are multi cultural countries and there are always migrants
Table 5: Race description inaccuracy

\begin{tabular}{|c|c|c|c|c|}
\hline Category & Factor & $\begin{array}{l}\text { Factor } \\
\text { count }\end{array}$ & $\begin{array}{l}\text { Total factor } \\
\text { count }\end{array}$ & $\begin{array}{l}\text { Factor } \\
\text { count }(\%)\end{array}$ \\
\hline Race & American & 161 & 3391 & 4.7 \\
\hline Race & Asian & 221 & 3391 & 6.5 \\
\hline Race & Australia & 7 & 3391 & 0.2 \\
\hline Race & Belgium & 8 & 3391 & 0.2 \\
\hline Race & Black & 721 & 3391 & 21.3 \\
\hline Race & Caucasian & 170 & 3391 & 5.0 \\
\hline Race & Celtic & 1 & 3391 & 2.9 \\
\hline Race & Chinese & 122 & 3391 & 3.6 \\
\hline Race & Colombia & 24 & 3391 & 0.7 \\
\hline Race & Denmark & 42 & 3391 & 1.2 \\
\hline Race & Europe & 12 & 3391 & 0.4 \\
\hline Race & France & 10 & 3391 & 0.3 \\
\hline Race & Germany & 46 & 3391 & 1.4 \\
\hline Race & Hispanic & 240 & 3391 & 7.0 \\
\hline Race & Indian & 79 & 3391 & 2.3 \\
\hline Race & Israel & 2 & 3391 & 5.9 \\
\hline Race & Italy & 184 & 3391 & 5.4 \\
\hline Race & Japan & 38 & 3391 & 1.1 \\
\hline Race & Korean & 38 & 3391 & 1.1 \\
\hline Race & Malay & 1 & 3391 & 2.9 \\
\hline Race & Mexican & 10 & 3391 & 0.3 \\
\hline Race & Mongoloid & 19 & 3391 & 0.6 \\
\hline Race & Netherlands & 44 & 3391 & 1.3 \\
\hline Race & New Zealand & 14 & 3391 & 0.4 \\
\hline Race & Non black & 11 & 3391 & 0.3 \\
\hline Race & Oriental & 19 & 3391 & 0.6 \\
\hline Race & Panama & 30 & 3391 & 0.9 \\
\hline Race & Philippines & 24 & 3391 & 0.7 \\
\hline Race & Scandinavian & 36 & 3391 & 1.0 \\
\hline Race & South Africa & 45 & 3391 & 1.3 \\
\hline Race & Spain & 45 & 3391 & 1.3 \\
\hline Race & Switzerland & 10 & 3391 & 0.3 \\
\hline Race & Taiwan & 18 & 3391 & 0.5 \\
\hline Race & Texas & 15 & 3391 & 0.4 \\
\hline Race & Turkey & 27 & 3391 & 0.8 \\
\hline Race & UK & 56 & 3391 & 1.7 \\
\hline Race & White & 841 & 3391 & 25.0 \\
\hline
\end{tabular}

to these countries. This kind of data might be confusing and misleading to a point because race, lifestyle, type of food, type of work are all factors that can affect the occurrence of adverse drug reactions. When a study mentions the race Asian, Asian includes Chinese, some of the Arabs, Malays and Indians. They are all different people with different life styles and different climates.

Some races appear to be more vulnerable to adverse drug reactions than others. A study on epidemiological risk factors for hypersensitivity reactions to abacavir found the Caucasian race as a risk factor for reactions. In a recent cohort study evaluating risk factors for adverse drug reactions associated with Angiotensin-Converting Enzyme (ACE) inhibitors, two thousand two hundred and twenty five (2225) people were evaluated. Of these $19 \%$ had to discontinue therapy due to adverse drug reactions, African Americans were found to be more susceptible to developing ACE-related angioedema than other ethnic groups $^{12]}$. 
Some ethnic groups may be more susceptible to adverse reactions during treatment with drugs than others. The risk of angioedema with antihypertensive medications was found to be three times greater in black patients than non-black patients. The risk of cough was also nearly three times higher in East Asian patients compared with white patients ${ }^{[13]}$.

Ethnicity is an important demographic variable contributing to inter-individual variability in drug metabolism and response. Some studies discuss the issue that genetic factors can determine individual susceptibility to both dose-dependent and doseindependent adverse drug reactions. Determinants of susceptibility include kinetic factors, such as gene polymorphisms in cytochrome P450 enzymes and dynamic factors, such as polymorphisms in drug targets. The relative importance of these factors will depend on the nature of the adverse drug reactions, however, it is likely that more than one gene will be involved in most instances ${ }^{[14]}$.

The races mentioned in the studies are all related to the countries not the original race. For example when the United Kingdom is mentioned, this does not mean Caucasians, they might be Black, Asian or any other race since the UK is a multi-cultural country. Specifications are of value in these issues.

Because of the importance of race in the medical decision process, the need for new regulatory approaches to medicines with race-specific indications is growing ${ }^{[15]}$.

\section{Lack of classification standards}

Age classification, race classification: Lack of specific age classification is obvious all over the literature, each article uses different age ranges, some mention age between $20-70$ years, others $40-80$, or it may be mentioned as $>40$ or $<60$. Many examples are available in Table 4. Age classification is important and needed in order to use studies outcomes in proper way.

There are no clear rules to control providing the exact race for every study done on patients, Table 5 shows many examples in this regard. There must be a standard to be adopted by medical journals to control the type of data that must be provided by each researcher.

ADR description inaccuracy: Ex. Smoking. It is not known if patients who developed the ADR were the smokers or not. The study mentions a certain number of people were smokers but they do not mention if those who develop the ADR were the smokers or non smokers. Another issue regarding adverse drug reaction description inaccuracy is that it is not mentioned if the side effects happened in the same patients or how many patients have more than one side effect. They only mention the percentage of ADR occurrence in general. This data is important and needs to be specified because it clarifies the type of patients who developed the ADR.

Out of the 15718 data collected and used in this study, nothing is mentioned about the occurrence of more than one ADR for the same patient. All literature mentions that a certain number of patients have the ADRs but because of the omitted data it is impossible to determine if the same people developed more than one ADRs or only one ADR was present in each patient. For example, if the study mentions that a certain percentage of patients developed an ADR, it is not mentioned if these ADRs occurred in different patients, or all in the same patient, i.e. did each patient have one ADR (e.g., nausea, headache or diarrhea) or did each patient have all three ADRs. This data is critical and it enables good medical decisions to be made and assists researchers using literature in a more effective manner.

\section{CONCLUSION}

Age, gender, race, smoking, health status, medication use and any other information related to the patients is basic information which needs to be available in all literature, not just to be mentioned in the methodology and sampling, but to be specified carefully and fully in the results.

Sometimes when more than one adverse drug reaction is mentioned in a study, the study fails to mention if those reactions are found in different patients or the same patient i.e., whether the patient has only one adverse drug reaction or more than one.

It is recommended that all of the following information must be available for every study related to drug safety and efficacy in order to be published.

Specific details about peoples enrolled in the research including their race, exact age, gender, medical diseases and anything which might affect a medical decision.

People's biodata and medical data should be related to the presence of adverse drug reactions, it is not enough to mention that a certain number of patients have ADRs, it is important to give details regarding those people so that the exact results are clear and precise.

It is recommended that the science of ethnicity should be adopted during the research in order to avoid mentioning the name of the countries where the study is done, this avoids omitting data related to race. Standards should be considered for not accepting any 
study that does not follow these measures and this is in order to unify the criteria of all literature.

In order to publish an article it should contain certain data, without which the study will not be published. For example, a study that does not include gender or does not mention gender should not be accepted until the data is provided.

Some statistics must be provided by the study and should not be hidden in order to enable readers to utilize it in a clinical aspect.

\section{REFERENCES}

1. Ofotokun, I. and C. Pomeroy, 2003. Sex differences in adverse reactions to antiretroviral drugs. Top HIV Med, 11: 55-59. https://www. iasusa.org/pub/topics/2003/issue2/55.pdf.

2. Singh, N.P., M. Uppal, S. Anuradha, A. Agarwal and S.N. Rizvi, 1998. Angiotensin converting enzyme inhibitors and cough-a north Indian study. J. Assoc. Physicians India, 46: 448-451. http://www.ncbi.nlm.nih.gov/pubmed/11273288.

3. Budnitz Daniel, S., Shehab Nadine, R. Kegler Scott and L. Richards Chesley, 2007. Medication use leading to emergency department visits for adverse drug events in older adults. Ann. Internal Med., 147: 755-765. http://annals.highwire.org/cgi/ content/full/147/11/755.

4. Hajar, E.R. et al., 2003. Adverse drug reaction risk factors in older outpatients. Am. J. Geriatric Pharmacotherapy. 1: 82-89. doi:10.1016/S15435946(03)90004-3.

5. Jimmy Jose and G.M. Padma Rao, 2006. Pattern of adverse drug reactions notified by spontaneous reporting in an Indian tertiary care teaching hospital. Pharmacol. Res., 54: 226-233. Doi: 10.1016/J.Phrs.2006.05.003.

6. Martin, R.M., P.N. Biswas, S.N. Freemantle, G.L. Pearce and R.D. Mann, 1998. Age and sex distribution of suspected adverse drug reactions to newly marketed drugs in general practice in England: analysis of 48 cohort. Br. J. Clin. Pharmacol., 46: 505-511. Doi: 10.1046/j.13652125.1998.00817.x.

7. Gurwitz, J.H. et al., 2003. Incidence and preventability of adverse drug events among older persons in the ambulatory setting. JAMA, 289: 1107-1116. http://jama.amaassn.org/cgi/content/abstract/289/9/1107.
8. Mish, F.C., 2003. Merriam-Webster's Collegiate Dictionary. 11th Edn. Springfield, Mass: MerriamWebster, http://www.merriam-webster.com/info/ znew_words.htm.

9. Margaret A. Winker, 2004. Measuring race and ethnicity: Why and how? JAMA, 292: 1612-1614. http://jama.ama-assn.org/cgi/content/full/292/13/ 1612.

10. Risch, N., E. Burchard, E. Ziv and H. Tang, 2002. Categorization of humans in biomedical research: Genes, race and disease. Genome Biol., 3. Doi: 10.1186/gb-2002-3-7.

11. McDowell, S.E., J.J. Coleman and R.E. Ferner, 2006. Systematic review and meta-analysis of ethnic differences in risks of adverse reactions to drugs used in cardiovascular medicine. BMJ, 332: 1177-1181. doi:10.1136/bmj.38803.528113. 55.

12. Morimoto, T. et al., 2004. An evaluation of risk factors for adverse drug events associated with angiotensin-converting enzyme inhibitors. J. Eval. Clin. Pract., 10: 499-509. http://www.ncbi.nlm.nih. gov/pubmed/15482412.

13. Coleman, J.J., R.E. Ferner, A.H. Jacqueline and S.E. McDowell, 2006. Do adverse drug reactions to cardiovascular medicines differ among ethnic groups? A viewpoint. Medscape Family Medicine. Systematic Review and Meta-analysis of Ethnic Differences in Risks of Adverse Reactions to Drugs Used in Cardiovascular Medicine. BMJ. 332: 1177-1181. http://www.medscape.com/ viewarticle/540216.

14. Pirmohamed, M. and B.K. Park, 2001. Genetic susceptibility to adverse drug reactions. Trends Pharmacol. Sci., 22: 298-305. doi:10.1016/S01656147(00)01717-X.

15. Winickoff David E. and K. Obasogie Osagie, 2008. Race-specific drugs: regulatory trends and public policy. Trends Pharmacol. Sci., 29: 277-279. doi:10.1016/j.tips.2008.03.008. 